Appl i cat i on of i nduced cur rent potent i al drop techni que for measur enent s of cracks on i nt er nal wal I of t ube- shaped speci mens

\begin{tabular}{|l|l|}
\hline 著者 & SATO Yasumbt o, ATSUM Takeo, SHOJI Tet suo \\
\hline $\begin{array}{l}\text { j our nal or } \\
\text { publ i cat i on ti t e }\end{array}$ & Journal of Test i ng and Eval uat i on \\
\hline vol une & 40 \\
\hline number & 7 \\
\hline page range & $497-504$ \\
\hline year & $2007-10$ \\
\hline URL & ht t p: //hdl . handl e. net /10098/1168 \\
\hline
\end{tabular}




\title{
Application of induced current potential drop technique for measurements of cracks on internal wall of tube-shaped specimens
}

Yasumoto Sato a*, Takeo Atsumi ${ }^{b}$, Tetsuo Shoji ${ }^{b}$

a: Graduate School of Engineering, Fukui University, 3-9-1 Bunkyo, Fukui 910-8507, Japan

b: Fracture and Reliability Research Institute, Tohoku University, 6-6-01 Aramaki Aoba, Aoba-ku Sendai 980-8579, Japan

* Corresponding author. Tel.: +81-776-27-9728; Fax: +81-776-27-9764.

E-mail address: yasumoto.sato@npes.fukui-u.ac.jp

\begin{abstract}
This paper presents the applicability of induced current potential drop (ICPD) technique to the detection and sizing of defects in objects with a shape of real components. The shape of tube was selected as one of the shape of real components and an ICPD sensor for the inspection of tube-shaped specimens was developed. The usefulness of the developed sensor was verified by experiments. Finite element analysis (FEA) was conducted to obtain calibration data to convert PD data measured using the sensor into the depth of defects. It was shown that the crack depths estimated based on measured potential drops and FEA showed good agreement with the crack depths estimated by ultrasonic testing.
\end{abstract}

Key words:

Nondestructive evaluation, Thermal fatigue crack, Tube-shaped specimen, Finite element analysis, Potential drop 


\section{Introduction}

Depth estimation of defects is conducted when defect is detected in an in-service inspection to investigate whether the structural integrity of the components and/or the plants is affected by the existence of the defect. In many cases, ultrasonic testing has been utilized for the depth estimation. However, the depth estimation by the ultrasonic testing requires the technical skill of operator, and hence nondestructive methods which permit the detection and sizing of defects without the skill of operator is required. The signals of ultrasonic testing are sensitive to the macroscopic structure of the material under measurement, and this is one of the causes that the ultrasonic testing requires the technical skill from operators. In contrast, the signals of electromagnetic nondestructive testing methods are affected by more macroscopic structures [1].

One of the electromagnetic nondestructive methods, potential drop (PD) techniques have been utilized for the detection and sizing of defects in conductive materials. The PD techniques are divided roughly into a direct current potential drop (DCPD) technique [2] and an alternating current potential drop (ACPD) technique [3] according to the current utilized. The PD techniques permit precise measurement of defect depth without the technical skill. However, usual PD techniques need terminals to supply a current to the material under measurement, and thus the PD techniques are unsuitable for inspection. Consequently, the PD techniques have mainly been utilized for monitoring of crack initiation and propagation in laboratories [4, 5]. One of the ACPD techniques, namely the induced current potential drop (ICPD) technique, based on electromagnetic induction, has been developed [6] to overcome the difficulty of inspection of the PD techniques. The usefulness of the ICPD technique for the detection and sizing of defects in simple specimens such as plates and/or round-bars [7-10] has been demonstrated. However, the applicability of the ICPD technique to the detection and sizing of defects in objects with a shape of real components has not been investigated. Further, in order to convert PD data into defect depths, the ICPD technique requires calibration data measured on the specimen of a similar material and a shape to the objects under inspection, and this fact has made the ICPD technique unsuitable for the inspection of objects with a shape of real components.

For the purpose of investigating the applicability of the ICPD technique to the detection and sizing of cracks in objects with a shape of real components, we selected a tube shape as one of the shape of real components. Then a sensor for cracks on an internal wall of tube-shaped objects was developed and depth estimation was performed combined with finite element analysis.

2. Development of a sensor for cracks on the internal wall of tube-shaped specimens

2.1 Sensor for the ICPD technique and typical signal distribution for surface breaking cracks

The ICPD technique supplies an alternating current to the object under inspection using induction coil located near the surface of the object, without direct contact, unlike the standard PD techniques in which a current is directly supplied to the object under inspection through electrical terminals, and measures the perturbation of the current which occurs as a result of defects. A typical ICPD sensor for a plate 
specimen and signal distribution measured using the sensor on a Ni-based alloy 600 plate containing an artificial surface breaking crack with a depth of 3mm are shown in Figures 1 [8] and 2, respectively. The horizontal axis of Figure 2 shows the position of the centre between the two electrical terminals for PD measurement whilst the vertical axis indicates the normalized potential drop (NPD), which represents the ratio of the measured $\mathrm{PD}$ to the average $\mathrm{PD}$ of an intact region. The maximum normalized potential drop $\left(V_{\max }^{-}\right.$or $V_{\max }^{+}$in Figure 2$)$ should be used as a representative NPD (R-NPD) for the measurement. However, it is difficult to measure this value accurately due to its strong dependence on the position of the sensor with respect to the defect. Consequently, in this study, the normalized potential drop measured just above the defect ( $V$ c in Figure 2 ) is used as a R-NPD for the measurement because it is relatively easy to measure its exact value.

\subsection{The ICPD sensor for cracks on the internal wall of tube-shaped specimen}

The target defects in this study are axial and circumferential cracks on the internal wall of tube-shaped specimens. As mentioned above, the ICPD sensor contains an induction coil. The coil utilized in the developed sensor for tube-shaped specimen is tilted in 45 degrees to the axial direction of the specimen to induce a current with components of axial and circumferential directions. Potential drops are measured using individual PD pick-up pins for both axial and circumferential cracks. A schematic of the developed sensor and a photo of the sensor are shown in Figures 3 and 4, respectively.

\section{Verification measurements}

\subsection{Tube-shaped specimen}

The applicability of the developed sensor to the inspection of cracks on the internal wall of tube-shaped objects was verified by performing measurements of fatigue cracks in the specimen. The specimen used for the verification measurements has a shape of tube with an outer diameter of $100 \mathrm{~mm}$, an inner diameter of $70 \mathrm{~mm}$ and a length of $300 \mathrm{~mm}$ (Figure 5). Three axial cracks and one circumferential crack were introduced on the internal wall of the specimen by thermal fatigue [11]. The locations of the cracks on the internal wall of the specimen are unknown. Directions and sizes of the cracks in the specimen are summarized in Table 1.

\subsection{Measuring system}

A schematic of the measuring system is shown in Figure 6. The system contains a sensor, a measurement instrument and a specimen. The measurement instrument is a commercial available alternating current (AC) potential drop measurement apparatus with a stable AC power source, a phase detector and a digital voltage meter.

\subsection{Measurement procedure}

At the beginning of measurements, course measurement was performed to identify crack locations, 
and fine measurement was then performed around the identified crack locations. For the fine measurement, measuring lines parallel to the axial direction of the specimen (X-direction shown in Figure 5) were set every $2 \mathrm{~mm}$ interval in circumferential direction. Potential drop maps were made around the detected cracks by scanning the sensor with $2 \mathrm{~mm}$ interval along the measuring line.

Alternating current supplied to the induction coil was set to $3 \mathrm{kHz}$ and 2 amperes. These values were defined by preliminary measurements.

\subsection{Results and discussion}

\subsubsection{Potential drop distributions}

Potential drop maps measured by the developed sensor around the cracks are shown in Figure 7 . Measured potential drops were normalized by the initial potential drop for each measuring line (Normalized potential drop; NPD). Higher NPDs are seen in the maps for each crack, and when examining a shape of potential drop distribution in perpendicular direction to the crack length, the shapes of the graphs presented in Figure 7 are similar to that shown in Figure 2. Accordingly, cracks on the internal wall of tube-shaped specimens can be detected by the developed sensor.

For the purpose of depth estimation, the maximum $V$ c, which is defined in Figure 2, was selected as R-NPD for each crack. The R-NPDs for the cracks are summarized in Table 2. The R-NPDs for cracks nos. 1, 2, and 3 that are similar depth are similar value and for deeper crack shows larger value.

4. Numerical simulation of potential drops for axial crack using finite element analysis

\subsection{Simulation conditions}

A schematic of a model analyzed by finite element analysis (FEA) is shown in Figure 8. The analyzed model contains a tube-shaped specimen, a tilted induction coil and air surrounding the specimen and the coil. An axial slit with semi-elliptical shape is introduced on the internal wall of the specimen as an axial crack using elements with same electromagnetic properties of air. The aspect ratio of the slit was approximately 0.3. The model containing only axial slit was analyzed in this study. The specimen was divided into 5 elements in wall thickness direction, 30 elements in axial direction and 40 elements in circumferential direction. Total number of elements and nodes including those of air were 39200 and 162 261, respectively. Finite element mesh of the specimen and the coil is presented in Figure 9. The electromagnetic properties used for the FEA are listed in Table 3.

\subsection{FEA results and discussion}

\subsubsection{Induced current density distribution}

Induced current density distribution on the internal wall of the tube-shaped specimen without slit is shown in Figure 10. The current is focused near the induction coil. However, the current flows in the specimen with a tilting angle of $33^{\circ}$ though that of the induction coil is $45^{\circ}$. The induced current flow must make a closed loop in the specimen because no current input/output terminals are connected to the specimen. The induced current in the specimen can be divided into two portion, namely, axial portion 
and circumferential portion. The circumferential portion of the current can make the closed loop in the specimen. However, the axial portion of the current can not make the closed loop in axial direction, and therefore, the axial portion may change its direction to circumferential direction. Accordingly, the current induced in the tube-shaped specimen using the tilted coil will mainly flow in circumferential direction.

\subsubsection{Potential drop distribution and depth estimation by FEA}

Potential drops are measured using the ICPD sensor scanned on an object under measurement. Thus, in order to calculate potential drop distribution for the ICPD technique by FEA, each of potential drops has to be calculated using individual finite element mesh for all of the sensor position of the measurement, and therefore, the calculation requires a lot of time. To prevent the time consuming calculation, the FEA was performed under the condition in which the induction coil was fixed and only the potential drop pick-up pins were scanned along the measuring line. In this case, a single finite element mesh is needed.

Normalized potential drop distribution calculated by FEA using a tube-shaped model containing an axial slit with a depth of $4 \mathrm{~mm}$ is shown in Figure 11. The structure of potential drop distribution for the tube-shaped model calculated by FEA is similar to that shown in Figure 2.

Following the calculation of potential drop distribution, representative NPDs (R-NPDs) defined in section 2 were calculated for slits with depths of 2,4 and $6 \mathrm{~mm}$. Calculated R-NPDs are plotted against the slit depth in Figure 12. It can be seen from Figure 12 that R-NPD and slit depth $(d)$ has a liner relation as follows;

$$
\mathrm{R}-\mathrm{NPD}=0.0581 d+1
$$

As summarized in Table 4, R-NPDs measured by the developed sensor for each crack were converted into crack depths using the equation (1). The estimated crack depth show good agreement with the crack depth estimated by the ultrasonic testing.

Correspondingly, detection and sizing of cracks on internal wall of tube can be performed using the developed ICPD sensor and the relation between R-NPDs and slit depth obtained by FEA.

\section{Conclusions}

In order to investigate the applicability of Induced Current Potential Drop (ICPD) technique to detection and sizing of cracks in objects with a shape of real components we selected a shape of tube as that for a real component. Then a sensor for cracks on an internal wall of tube-shaped specimen was developed and depth estimation was performed combined with finite element analysis. The following conclusions can be reached from the results:

(1) The developed sensor containing tilted induction coil can detect all of axial and circumferential cracks introduced on the internal wall of the tube-shaped specimen. The minimum depth of crack investigated in this study is $2 \mathrm{~mm}$ for axial crack and $1.8 \mathrm{~mm}$ for circumferential crack.

(2) Crack depths estimated based on the measured representative normalized potential drop and 
finite element analysis showed good agreement with the crack depths estimated by ultrasonic testing.

\section{Acknowledgements}

A part of this work was supported by "International Cooperative Program for Education and Research", founded by The Japanese Ministry of Education, Culture, Sports, Science and Technology.

\section{References}

[1] Janousek L, Yusa N, Miya K. Utilization of two-directional AC current distribution for enhancing sizing ability of electromagnetic nondestructive testing methods. NDT E Int 2006; 39: $542-546$.

[2] Kanoh Y, Abe H. Proposal for nondestructive evaluation procedure of 3D crack shape by means of electrical potential method. Trans Jpn Soc Mech Eng 1992; 58 (547): 72-9.

[3] Collins R, Michael DH, Clark R. Measurement of crack depth in a transition weld using ACPD. Rev Prog Quant Nondestr Eval 1992; 11: 545-52.

[4] Thompson CD, Carey DM, Perazzo NL. Effects of hydrogen on electropotential monitoring of stress corrosion crack growth. In: Proceedings of the eighth international symposium on environmental degradation of materials in nuclear power systems—water reactors, 1997; 366-71.

[5] Andersson M, Persson C, Melin S. Experimental and numerical investigation of crack closure measurements with electrical potential drop technique. Int J Fatigue 2006; 28: 1059-1068.

[6] Shoji T, Kim H. Current status and future prospect of potential drop technique for nondestructive evaluation. Issue Series Title: Piping Eng 1996; 38 (1): 51-60.

[7] Yi Y, Shoji T. Measurement of Shape of 3-Dimensional Surface Crack Using ICFPD Technique. Trans Jpn Soc Mech Eng 1997; 63 (605): 68-72.

[8] Sato Y, SHOJI T. Evaluation of Back-Wall Fatigue Cracks by Means of Remotely Induced Current Potential Drop Technique and Its FEM Simulation. Trans Jpn Soc Mech Eng 2006; 72 (724): 1949-1954.

[9] Sato Y, Takeda Y, Shoji T. Nondestructive evaluation of fatigue and creep-fatigue damage by means of ICFPD technique. Fatigue Fract Eng Mater Struct 2001; 24(12): 885-93.

[10] Yamashita M, Tada S, Sato Y, Shoji T. Nondestructive evaluation of fatigue and creep-fatigue damage in $12 \% \mathrm{Cr}$ stainless steel by the induced current focusing potential drop technique. J Test Eval 2001; 29 (6): 544-555.

[11] Kemppainen M, Virkkunen I, Pitkänen J, Paussu R, Hänninen H. Advanced flaw production method for in-service inspection qualification mock-ups. Nucl Eng Des 2003; 224: 105-117. 
Table 1 Summary of cracks introduced in the tube-shaped specimen by thermal fatigue

Table 2 Summary of R-NPD measured on the tube-shaped specimen using the developed sensor.

Table 3 Electromagnetic properties used for the FEA

Table 4 Crack depth estimation using calibration curve calculated by FEA

Figure 1 Illustration of the sensor for the ICPD technique [8].

Figure 2 Typical potential drop distribution around an artificial surface breaking crack in the plate specimen.

Figure 3 Illustrations of the developed sensor for cracks on the internal wall of tube-shaped specimens (dimensions in $\mathrm{mm}$ ).

(a) Top view

(b) Side view

(c) A-A section view when insert the sensor into the specimen and move it in the specimen.

(d) A-A section view during measurements.

Figure 4 Photo of the developed ICPD sensor.

Figure 5 Illustration of tube-shaped specimen used for the verification measurements (dimensions in $\mathrm{mm})$. Locations of cracks are unknown.

Figure 6 Illustration of measuring system for the ICPD technique.

Figure 7 Normalized potential drop distributions measured on the tube-shaped specimen using the developed sensor.

(a) Crack 1 (Axial crack)

(b) Crack 2 (Axial crack)

(c) Crack 3 (Axial crack)

(d) Crack 4 (Circumferential crack)

Figure 8 Illustration of the model analyzed by FEA (dimensions in $\mathrm{mm}$ ).

Figure 9 Finite element mesh of the tube-shaped specimen containing an axial semi-elliptical slit.

Figure 10 Induced current density distribution in the specimen calculated by FEA (View from $\mathrm{Z}$ direction).

Figure 11 Normalized potential drop distribution around a slit with depth of $4 \mathrm{~mm}$ calculated by FEM.

Figure 12 Relation between R-NPD and axial slit depth. 
Table 1 Summary of cracks introduced in the tube-shaped specimen by thermal fatigue

\begin{tabular}{ccc}
\hline No. & Axial/Circ. & Crack depth $^{\text {a }}$ \\
\hline $1(2)$ & Axial & $2.0 \mathrm{~mm}$ \\
$2(1)$ & Axial & $2.0 \mathrm{~mm}$ \\
3 & Axial & $4.4 \mathrm{~mm}$ \\
4 & Circ. & $1.8 \mathrm{~mm}$ \\
\hline
\end{tabular}

a. Estimated by specimen fabricator with ultrasonic sensor

Table 2 Summary of R-NPD measured on the tube-shaped specimen using the developed sensor.

\begin{tabular}{cccc}
\hline No. & Axial/Circ. & Crack depth a & R-NPD \\
\hline 1 & Axial & $2.0 \mathrm{~mm}$ & 1.14 \\
2 & Axial & $2.0 \mathrm{~mm}$ & 1.13 \\
3 & Axial & $4.4 \mathrm{~mm}$ & 1.26 \\
4 & Circ. & $1.8 \mathrm{~mm}$ & 1.10
\end{tabular}

a: Estimated by specimen fabricator with ultrasonic sensor

Table 3 Electromagnetic properties used for the FEA

\begin{tabular}{lcc}
\hline \multicolumn{1}{c}{ Material } & $\varepsilon_{\mathrm{r}}{ }^{\mathrm{a}}$ & $\rho^{\mathrm{b}}$ \\
\hline Alloy600 (Specimen) & 1.0 & $135 \times 10^{-8} \Omega \mathrm{m}$ \\
Cupper (Induction coil) & 1.0 & - \\
Air & 1.0 & - \\
\hline${ }^{\mathrm{a}}: \varepsilon_{\mathrm{r}} \quad$ Relative permeability & & \\
b. $: \rho$ Electrical resistivity & &
\end{tabular}

Table 4 Crack depth estimation using calibration curve calculated by FEA

\begin{tabular}{cccccc}
\hline No. & Axial/Circ. & Crack depth $^{\text {a }}$ & R-NPD & Estimated crack depth & Difference \\
\hline 1 & Axial & $2.0 \mathrm{~mm}$ & 1.14 & $2.4 \mathrm{~mm}$ & $+0.4 \mathrm{~mm}$ \\
2 & Axial & $2.0 \mathrm{~mm}$ & 1.13 & $2.2 \mathrm{~mm}$ & $+0.2 \mathrm{~mm}$ \\
3 & Axial & $4.4 \mathrm{~mm}$ & 1.26 & $4.5 \mathrm{~mm}$ & $+0.1 \mathrm{~mm}$ \\
4 & Circ. & $1.8 \mathrm{~mm}$ & 1.10 & $1.7 \mathrm{~mm}$ & $-0.1 \mathrm{~mm}$ \\
\hline
\end{tabular}

a: Estimated by specimen fabricator with ultrasonic sensor 


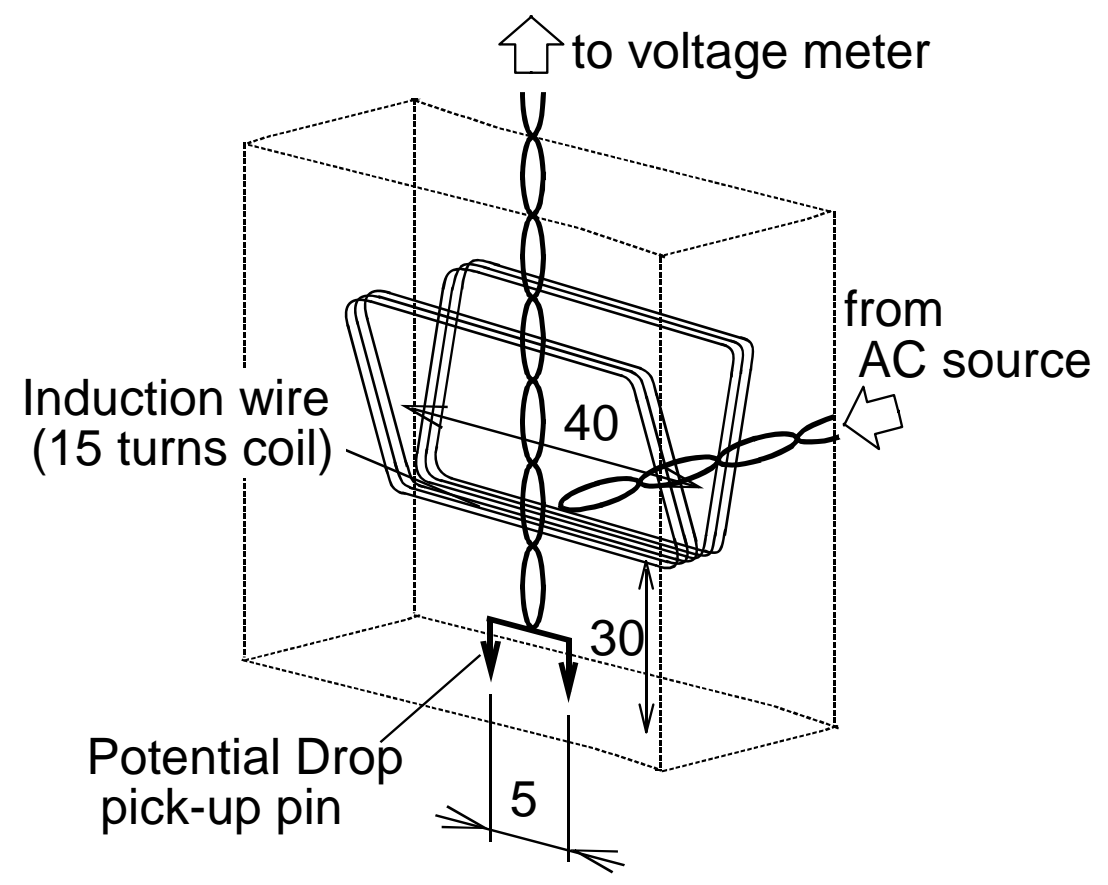

Figure 1 Illustration of the sensor for the ICPD technique [8].

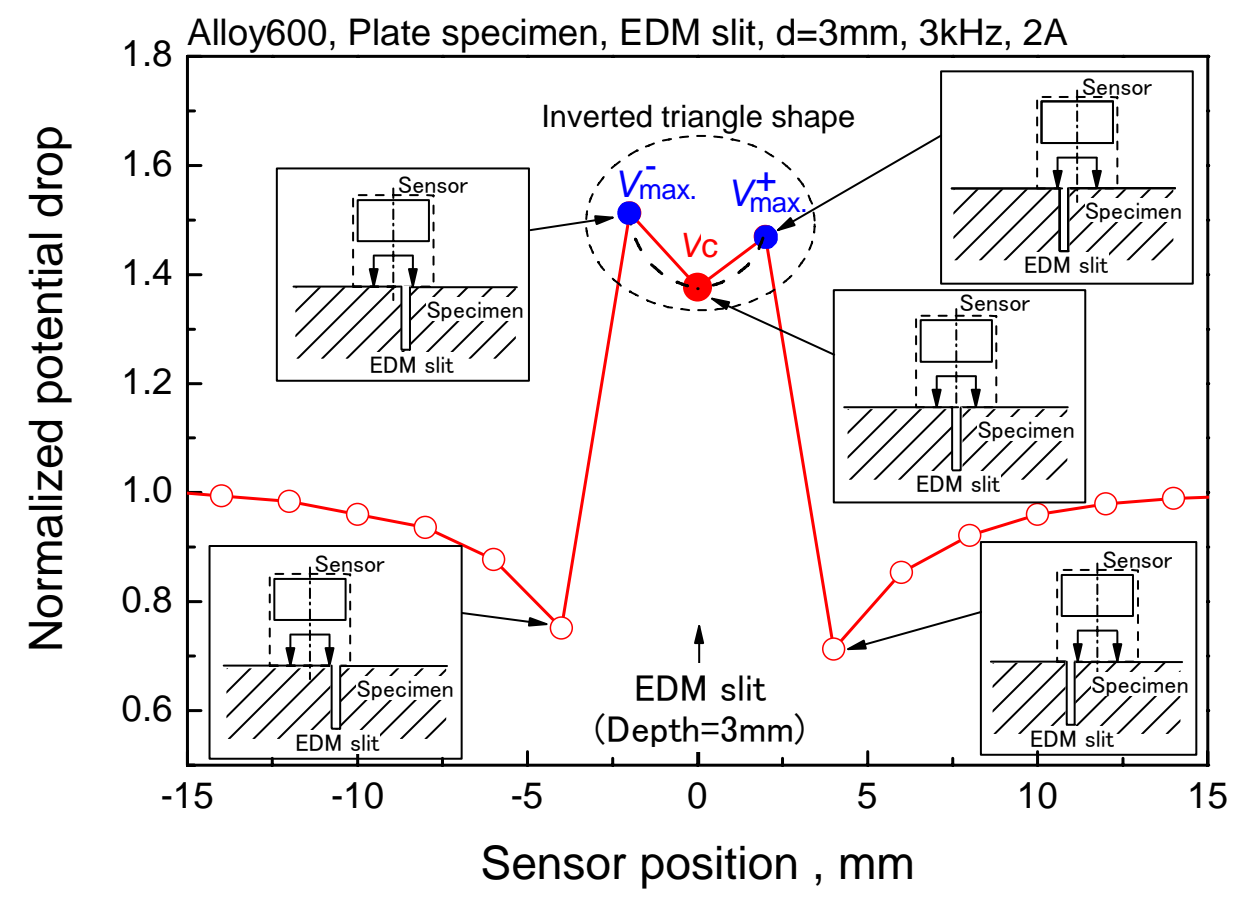

Figure 2 Typical potential drop distribution around an artificial surface breaking crack in the plate specimen. 
a

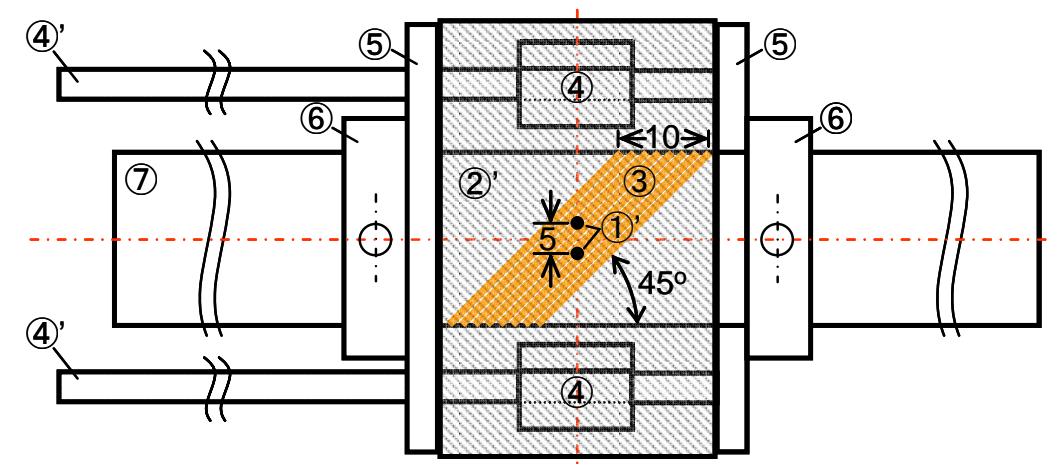

(1) Potential drop pick-up pins (for circ. cracks)

(1)' Potential drop pick-up pins (for axial cracks)

(2) Pin holder (for circ. cracks)

(2)' Pin holder (for axial cracks)

(3) Induction coil (24 turns)

(4) Pin holder drive cam

(4)' Pin holder drive camshaft

(5) Pin holder press board

(6) Holding ring

(7) Sensor core

b

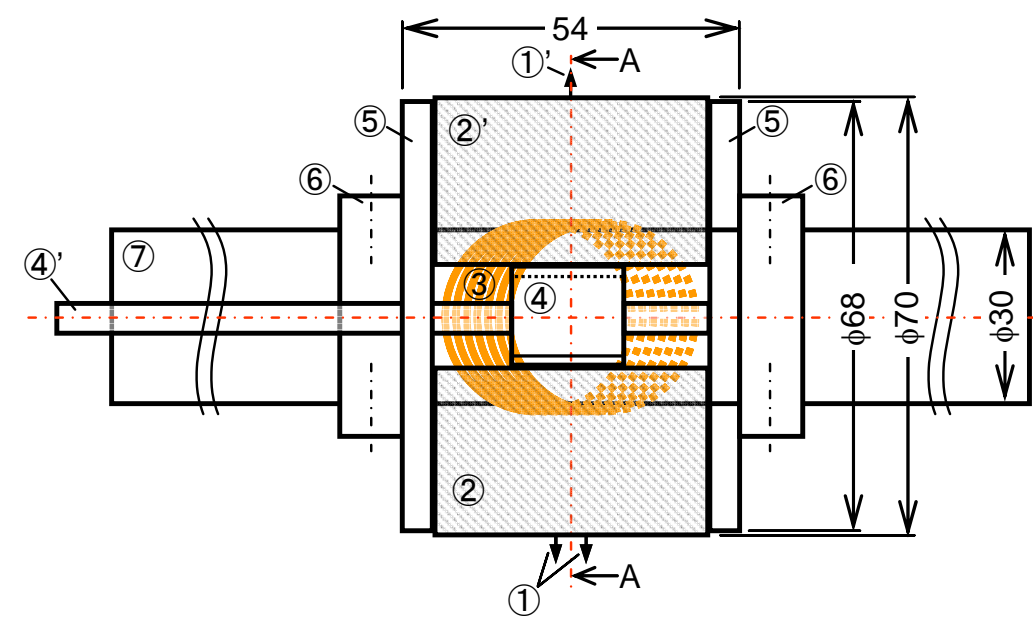

C
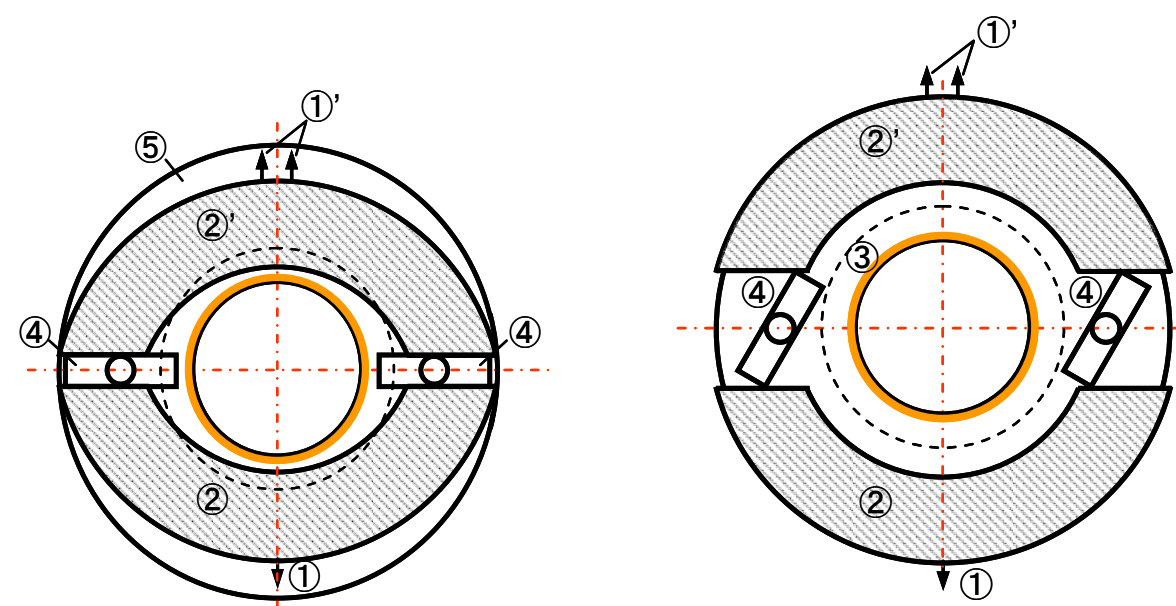

Figure 3 Illustrations of the developed sensor for cracks on the internal wall of tube-shaped specimens (dimensions in mm): (a) Top view, (b) Side view, (c) A-A section view when insert the sensor into the specimen and move it in the specimen, (d) A-A section view during measurements. 


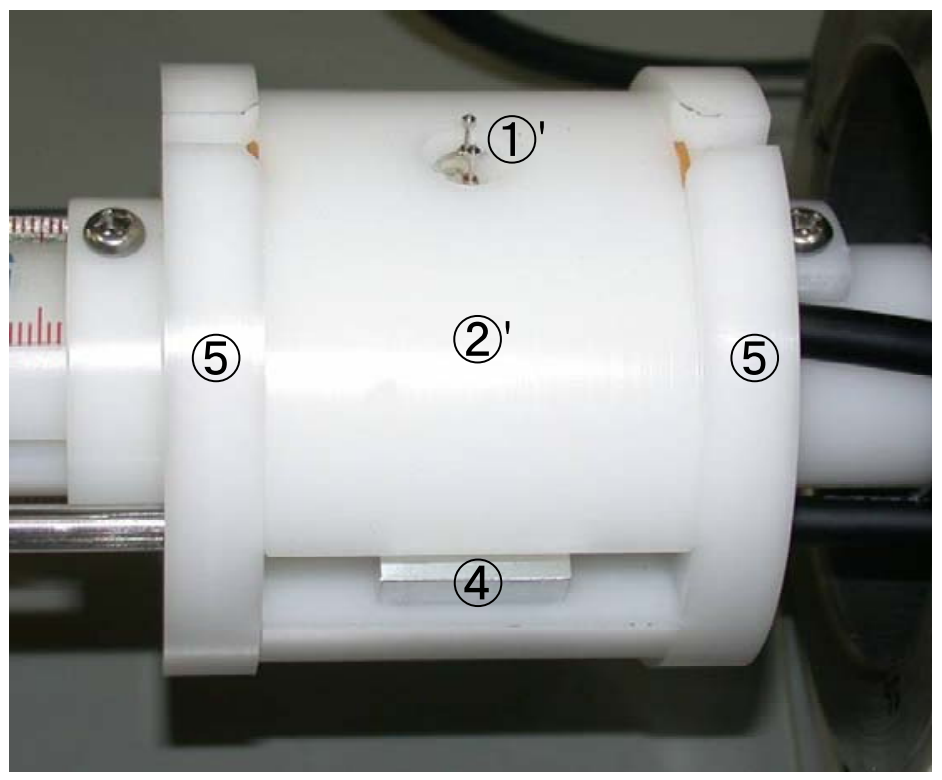

Figure 4 Photo of the developed ICPD sensor.

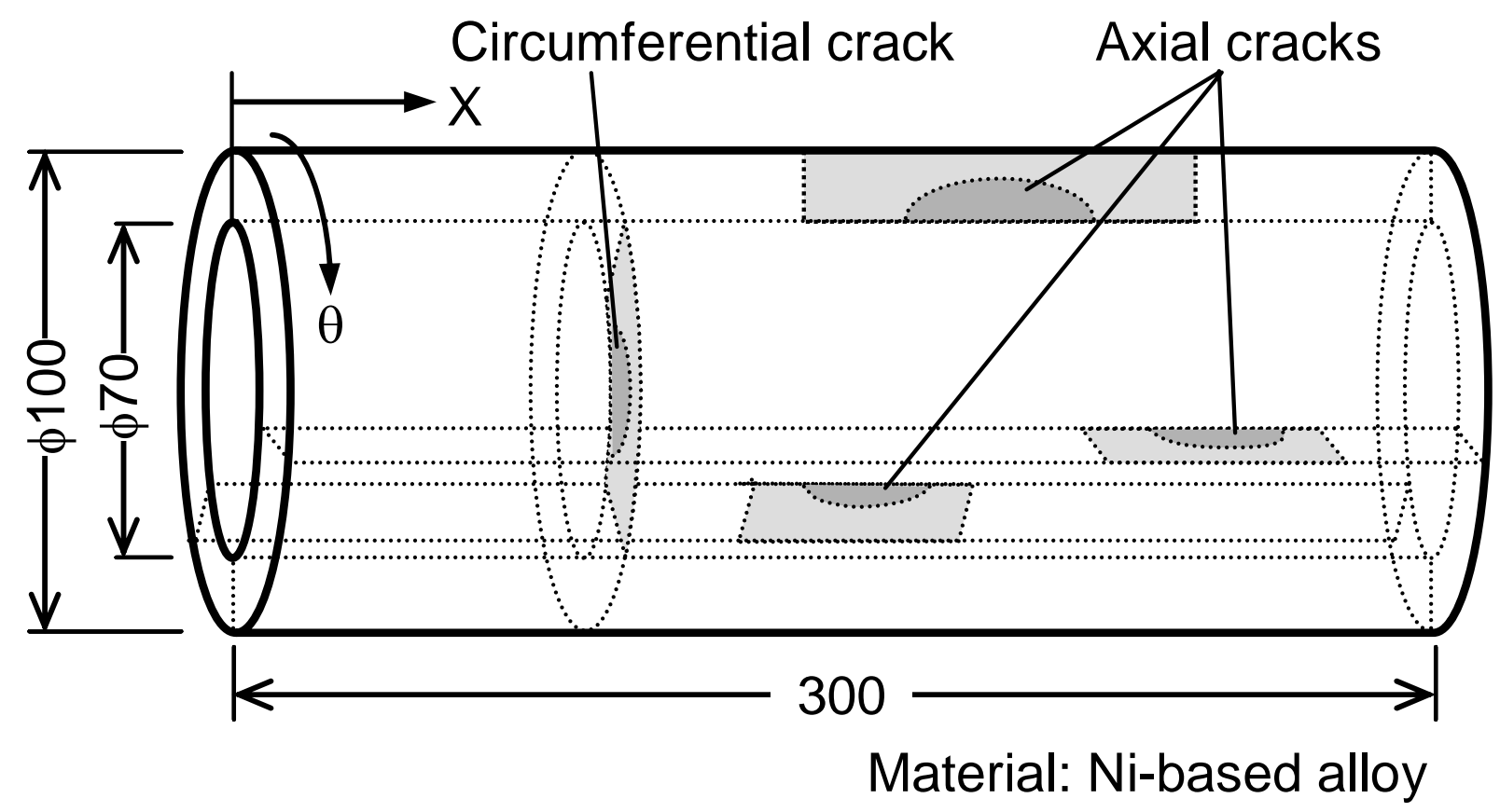

Figure 5 Illustration of tube-shaped specimen used for the verification measurements (dimensions in $\mathrm{mm})$. Locations of cracks are unknown. 


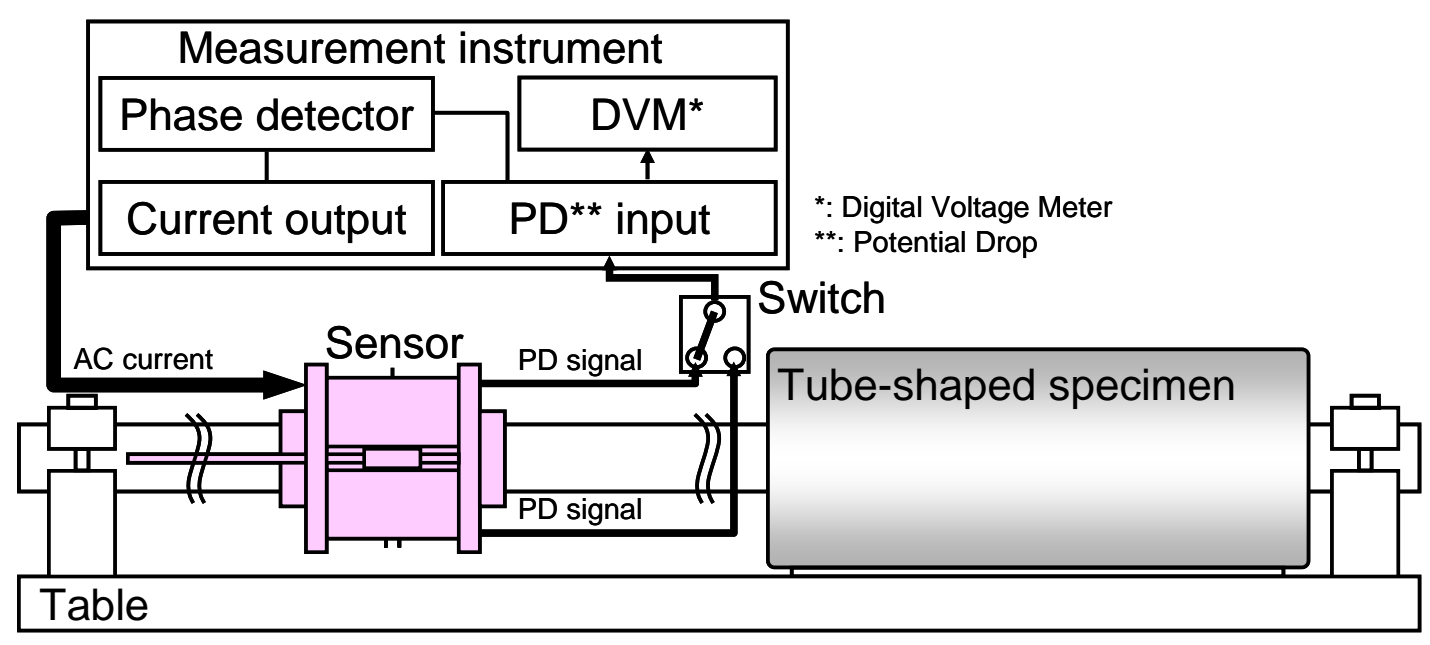

Figure 6 Illustration of measuring system for the ICPD technique.

a

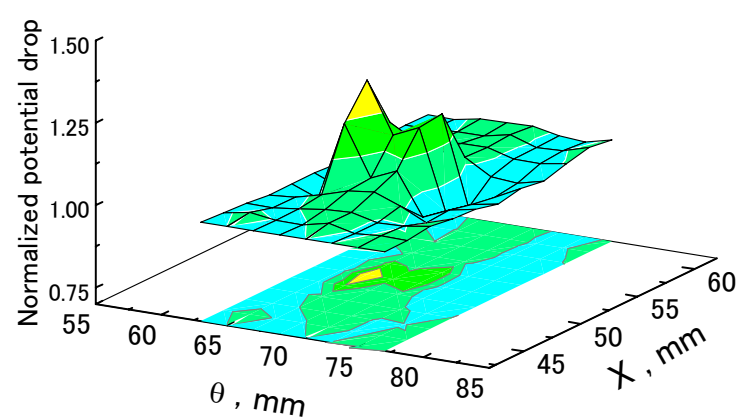

C

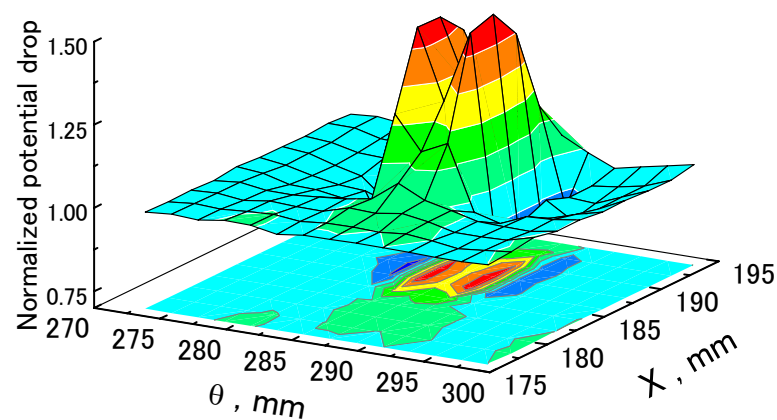

b

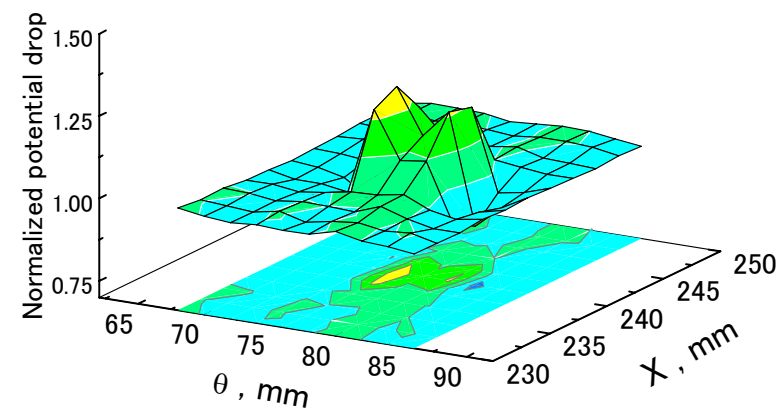

d

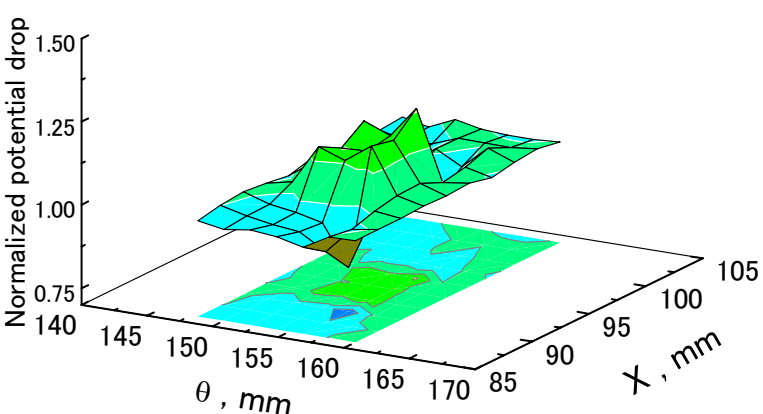

Figure 7 Normalized potential drop distributions measured on the tube-shaped specimen using the developed sensor: (a) Crack 1 (Axial crack), (b) Crack 2 (Axial crack), (c) Crack 3 (Axial crack), (d) Crack 4 (Circumferential crack) 


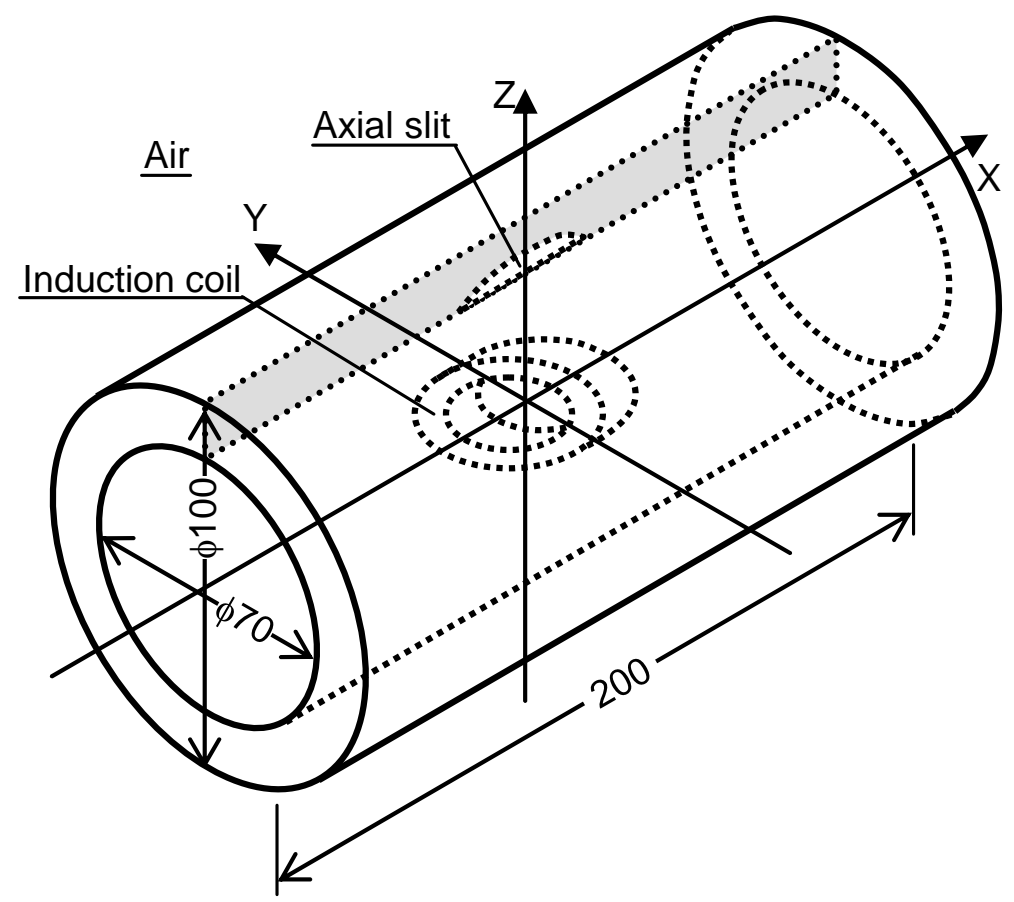

Figure 8 Illustration of the model analyzed by FEA (dimensions in $\mathrm{mm}$ ).

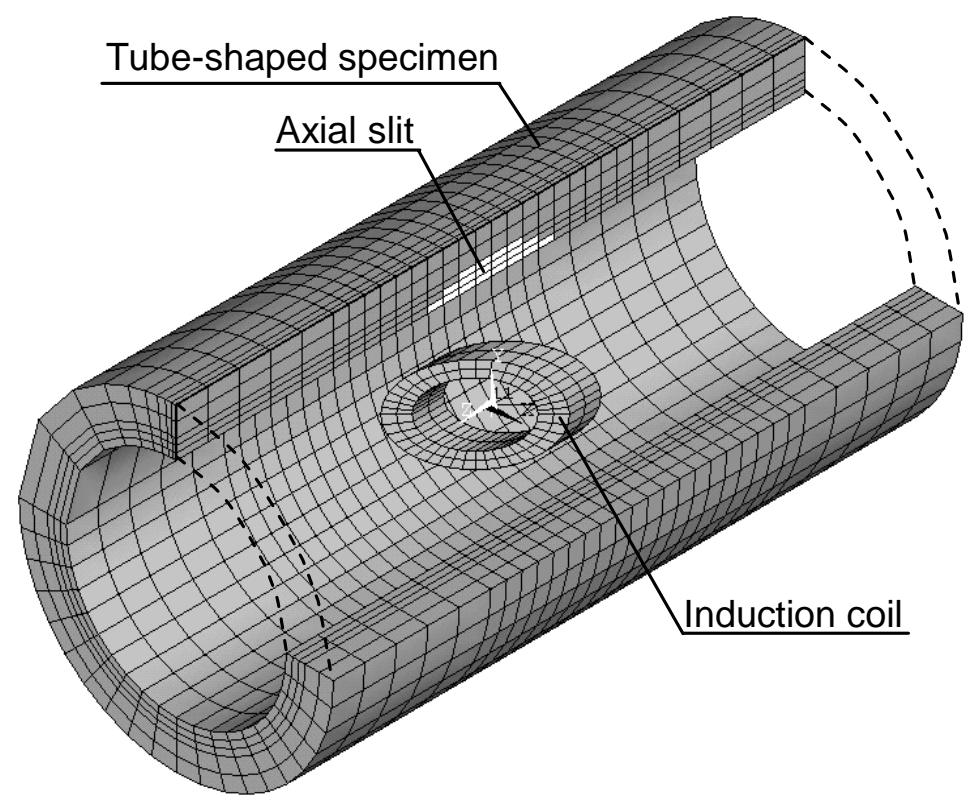

Figure 9 Finite element mesh of the tube-shaped specimen containing an axial semi-elliptical slit. 


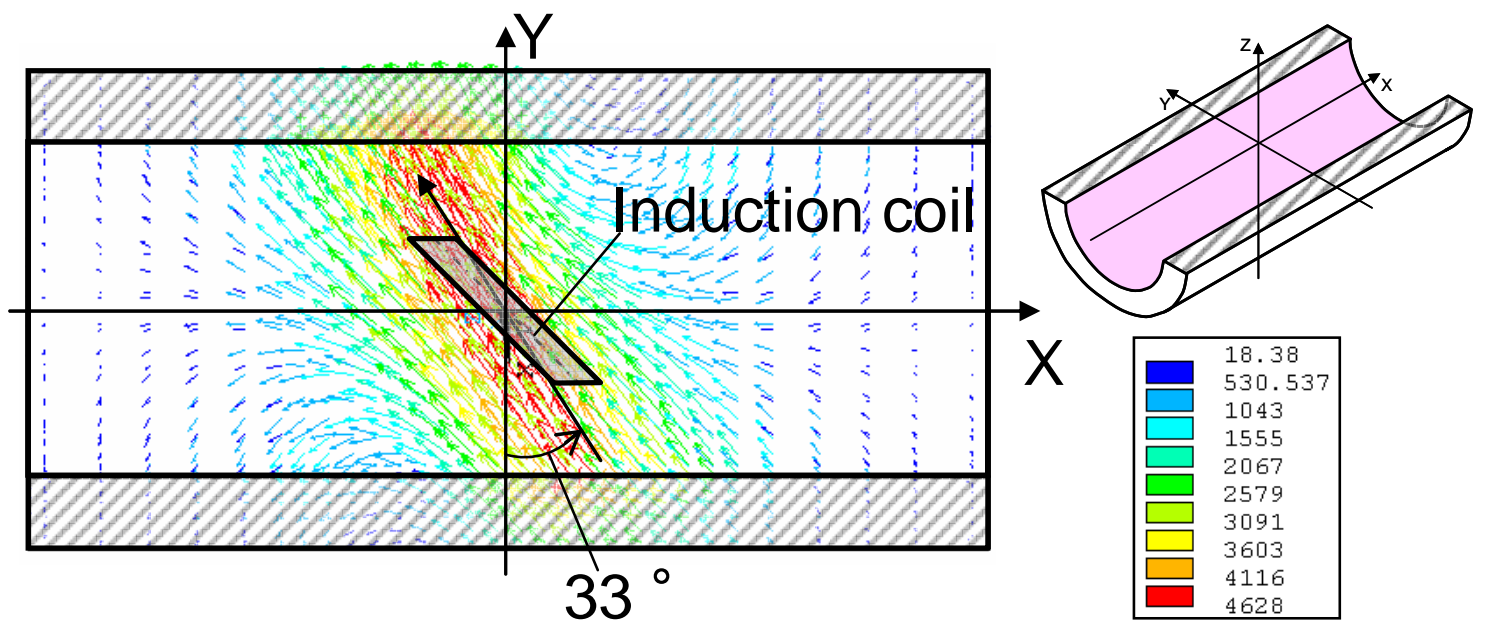

Figure 10 Induced current density distribution in the specimen calculated by FEA (View from Z direction).

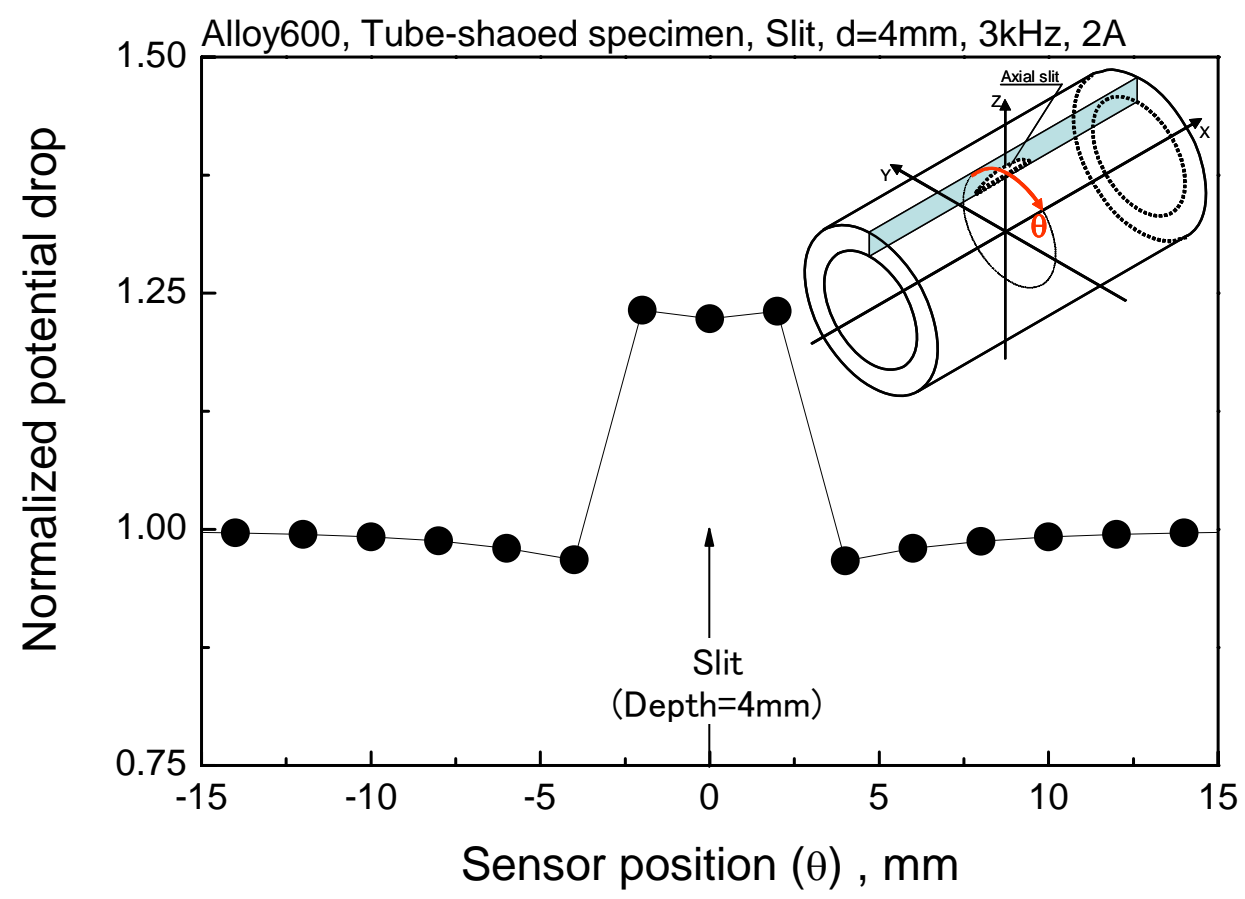

Figure 11 Normalized potential drop distribution around a slit with depth of $4 \mathrm{~mm}$ calculated by FEM. 


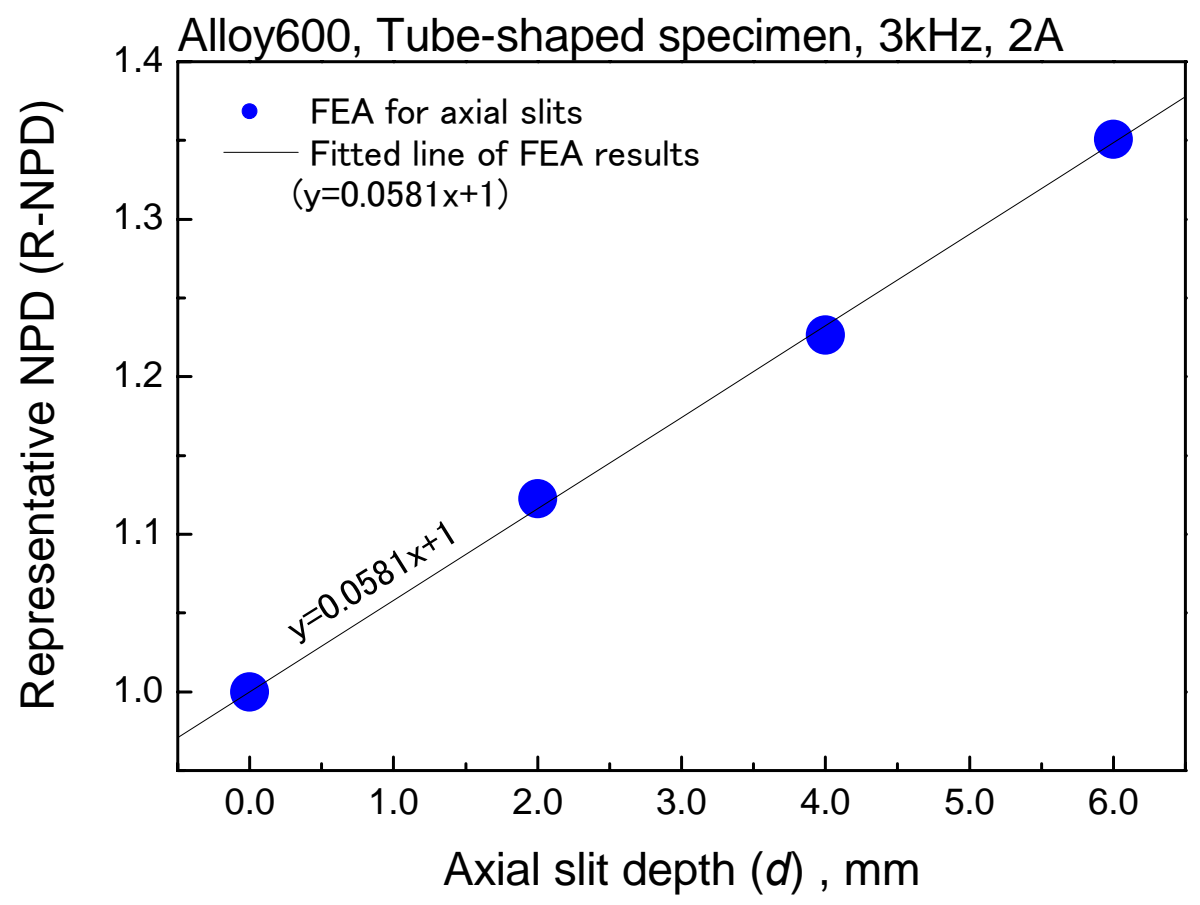

Figure 12 Relation between R-NPD and axial slit depth. 